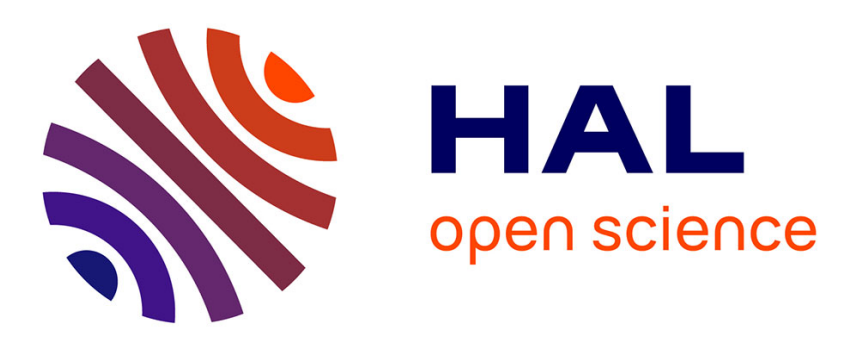

\title{
Wind Turbine Noise Modeling Based on Amiet's Theory: Effects of Wind Shear and Atmospheric Turbulence
}

\author{
Yuan Tian, Benjamin Cotté
}

\section{To cite this version:}

Yuan Tian, Benjamin Cotté. Wind Turbine Noise Modeling Based on Amiet's Theory: Effects of Wind Shear and Atmospheric Turbulence. Acta Acustica united with Acustica, 2016, 102 (4), pp.626-639. 10.3813/AAA.918979 . hal-01356102

HAL Id: hal-01356102

https://hal-ensta-paris.archives-ouvertes.fr/hal-01356102

Submitted on 24 Jan 2018

HAL is a multi-disciplinary open access archive for the deposit and dissemination of scientific research documents, whether they are published or not. The documents may come from teaching and research institutions in France or abroad, or from public or private research centers.
L'archive ouverte pluridisciplinaire HAL, est destinée au dépôt et à la diffusion de documents scientifiques de niveau recherche, publiés ou non, émanant des établissements d'enseignement et de recherche français ou étrangers, des laboratoires publics ou privés. 


\title{
Wind Turbine Noise Modeling Based on Amiet's Theory: Effects of Wind Shear and Atmospheric Turbulence
}

\author{
Y. Tian, B. Cotté \\ Institute of Mechanical Sciences and Industrial Applications (IMSIA), ENSTA ParisTech, CNRS, \\ CEA, EDF, Université Paris-Saclay, 828, bd des Maréchaux, 91762 Palaiseau cedex, France. \\ benjamin.cotte@ensta.fr
}

\begin{abstract}
Summary
Broadband noise generated aerodynamically is the dominant source for a modern wind turbine. In this paper, trailing edge noise and turbulent inflow noise are modeled using Amiet's theory to predict wind turbine noise spectra, directivity and amplitude modulation. First, by comparing model predictions with wind tunnel experiments from the literature, we show that a wall pressure spectral model that includes the effect of an adverse pressure gradient is needed to correctly predict trailing edge noise spectra. Then, we adapt the model to rotating blades and compare sound power level spectra of trailing edge noise with field measurements, assuming a constant wind speed profile. A good agreement is found at frequencies higher than approximately $1000 \mathrm{~Hz}$, but the levels are underestimated at lower frequencies. Finally, we account for wind shear and atmospheric turbulence effects using the Monin-Obukhov similarity theory. On the one hand, we show that angle of attack variations due to wind shear can produce a significant change in the wall pressure spectra of some blade sections, especially in stable atmospheric conditions, even though this effect is not clearly seen on the trailing edge noise spectra at the receiver. On the other hand, turbulent inflow noise does vary with atmospheric conditions, and contributes significantly to the noise radiated by a wind turbine at low frequencies. When both mechanisms are considered, the predicted sound power level spectra are in good agreement with measurements.
\end{abstract}

PACS no. 43.28.Ra, 43.50.Nm

\section{Introduction}

Wind turbine noise is one of the main concern for the acceptance of wind farms by the neighborhood [1]. For modern megawatt-sized wind turbines, it is generally admitted that broadband aerodynamic noise is dominant, with three main noise sources to consider [1, 2, 3]: turbulent inflow noise, trailing edge noise, and stall noise. Turbulent inflow noise is due to the interaction of atmospheric turbulence with the blade leading edge; its level depends on the strength of the turbulent fluctuations. Trailing edge noise is caused by the scattering of the turbulent boundary layer at the blade trailing edge; it is thus referred to as airfoil self-noise. When the blade angle of attack (AoA) increases, the boundary layer becomes partially separated and eventually complete separation or stall is achieved. This is a very intense noise mechanism called separation or stall noise.

Received $0 \quad 0$, accepted 00 .
A main feature of wind turbine noise is the amplitude modulation (AM), caused by the rotation of the blades, which is believed to be the most annoying feature of this noise source [1]. As explained in the project "Wind Turbine Amplitude Modulation" funded by RenewableUK [4, 3, 5], it is common to distinguish between normal amplitude modulation, also called swish, and enhanced or other amplitude modulation, also called thump. Normal AM corresponds to the sound level variation of a few decibels that modern wind turbines normally produce, mostly noticeable close to the turbine in the crosswind direction. On the other hand, enhanced AM is observed in the far-field (typically more than $600 \mathrm{~m}$ ), mostly in the downwind direction, and can reach $10 \mathrm{~dB}$ or more.

In order to accurately predict wind turbine noise, it is important to understand the role of atmospheric conditions. Wind shear is often cited as an important factor explaining sound pressure levels higher than expected and enhanced AM $[6,4]$. Under stable atmospheric conditions, typically at night, the wind speed increase from the bottom to the top of the rotor can 
be large, so the AoA changes significantly during one blade rotation. Furthermore, wind speed is usually small close to the ground when high wind shear is present, which means that the background noise, also called wind noise or vegetation noise [7], is low and will not mask efficiently wind turbine noise. Another important atmospheric parameter is the strength of turbulent velocity fluctuations, which mostly impacts turbulent inflow noise. This strength depends on the atmospheric conditions, and may also be strongly enhanced if a wind turbine is in the wake of another turbine $[3,5]$.

To better understand wind turbine noise and its associated amplitude modulation, it is thus important to propose a model that takes into account the main noise mechanisms and the influence of atmospheric conditions. Three main types of models have been proposed in the literature. First, a semi-empirical model has been built on the extensive measurements of airfoil self-noise made by Brooks et al. [8]. This model, sometimes called BPM model, has been applied to wind turbine noise with some success by Zhu et al. [9] and Oerlemans et al. [2]. It is however difficult to assess the validity limits of such a model, since it is based on measurements for a NACA 0012 airfoil that is not representative of the airfoils used in wind turbine applications.

Second, models based on acoustic analogies have been proposed. There have been a few attempts to apply the frequency-domain models proposed by Amiet for turbulent inflow noise [10] and trailing edge noise [11] to wind turbines, e.g. in the study of Glegg et al. [12]. Also, Lee et al. [13] recently proposed a trailing edge noise model based on the timedomain solution of the Ffowcs Williams-Hawkings equation [14]. Although these models are promising, they have not been thoroughly validated for wind turbine noise applications. In the case of Amiet's model for trailing edge noise, for example, one of the main difficulty is to have access to wall pressure statistics, whose modeling remains on open issue $[15,16,17,18]$.

Third, numerical tools of Computational Fluid Dynamics are getting more and more popular in the context of wind turbine noise. Reynolds Averaged NavierStokes (RANS) simulations can be used to provide information on the turbulent boundary layer developing over an airfoil. An approach consisting in coupling a RANS flow solver to the TNO-Blake model to predict the wall pressure spectrum has been proposed by several research groups [17, 18], and is able to capture adverse pressure gradient and turbulence anisotropy effects. Hybrid approaches for airfoil noise prediction based on Large Eddy Simulation (LES) have also been developed. For instance, Shen et al. [19] proposed a LES-based approach that consists in splitting the compressible flow problem into a viscous incompressible flow part and an inviscid acoustic part.
This approach has been applied to both symmetric and asymmetric airfoils [19, 17].

In this paper, we propose a physically-based wind turbine noise prediction model based on Amiet's theory in order to obtain accurate predictions in an efficient way. Both turbulent inflow noise and trailing edge noise are considered, and prediction results are validated against measurements from the literature. In order to account for wind shear and atmospheric turbulence effects, we use the Monin-Obukhov similarity theory that predicts wind speed profiles and turbulence parameters in the atmospheric surface layer. We focus in this paper on wind turbine noise close to the source (typically $100 \mathrm{~m}$ away), and study different phenomena such as directivity and amplitude modulation. Thus, in the following, the term amplitude modulation (AM) means normal AM. Although only near-field results are presented here, our longterm goal is to couple this source model to a propagation model in order to predict wind turbine noise at large distances (greater than $1 \mathrm{~km}$ ), and thus to better understand the possible causes of enhanced AM.

The paper is organized as follows. First, Section 2 presents Amiet's analytical model for turbulent inflow noise and trailing edge noise of a fixed airfoil and its validation against wind tunnel measurements. Then, in Section 3, we apply the trailing edge noise model to a full size wind turbine and compare predictions to field measurements considering a constant wind profile (no wind shear). Finally, the influence of wind shear and atmospheric turbulence on wind turbine noise is studied in Section 4 .

\section{Amiet's analytical model for turbu- lent inflow noise and trailing edge noise}

\subsection{Turbulent inflow noise}

\subsubsection{Original model for a fixed plate}

An airfoil in a turbulent flow experiences a fluctuating lift loading which will result in the generation of sound. Amiet derived airfoil response functions that relate the wavenumber spectrum of the incoming turbulence to the lift fluctuations over the blade surface [10]. These lift fluctuations can be seen as dipoles distributed along the airfoil surface that efficiently radiate noise to the far-field.

Amiet's model is based on linearized thin-airfoil theory, and the airfoil is reduced to a flat plate with zero thickness and zero angle of attack, with span $L$ and chord $c$, as seen in Figure 1. The flow is uniform with velocity $U$, and a receiver is placed in the farfield at $\left(x_{R}, y_{R}, z_{R}\right)$. The model is based on several assumptions:

1. the incoming turbulence fluctuation is considered to be small compared to the mean flow velocity; 


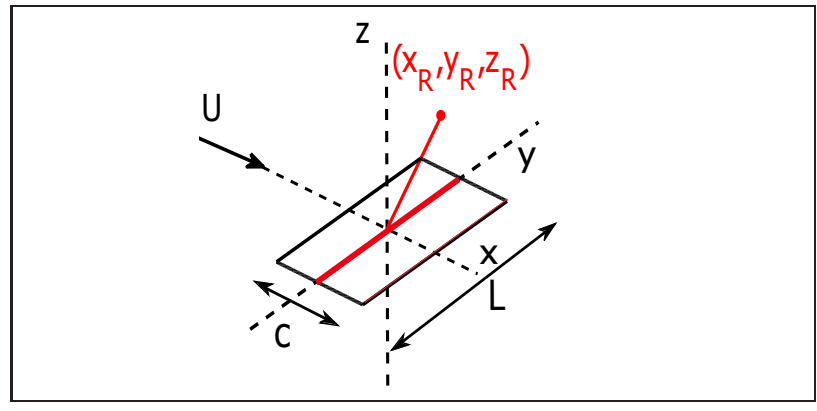

Figure 1: Schematics for Amiet's model applied to a fixed flat plate.

2. the interaction between the airfoil and the turbulent flow is inviscid so that the problem is reduced to solving linearized Euler equations;

3 . the turbulence is frozen, so that turbulent gust properties are unchanged while it is convected by the mean flow, and its velocity fluctuation is represented in terms of chordwise and spanwise wave numbers, here $K_{x}$ and $K_{y}$ respectively.

The problem can be described by a linearized Helmholtz equation associated with proper boundary conditions $[10,20]$, which form a Schwarzschild problem that can be solved analytically. For large aspect ratio, that is $L / c>3$, the far-field power spectral density (PSD) of acoustic pressure $S_{p p}$ is given by [10, 20]:

$$
\begin{gathered}
S_{p p}\left(x_{R}, y_{R}, z_{R}, \omega\right)=\left(\frac{\rho_{0} k c z_{R}}{2 S_{0}^{2}}\right)^{2} \pi U \frac{L}{2} \\
\Phi_{w w}\left(\frac{\omega}{U}, \frac{k y_{R}}{S_{0}}\right)\left|\mathscr{L}_{T I}\left(x_{R}, \frac{\omega}{U}, \frac{k y_{R}}{S_{0}}\right)\right|^{2},
\end{gathered}
$$

where $\omega$ is the angular frequency, $k=\omega / c_{0}$ is the acoustic wavenumber, $\rho_{0}$ is the air density, $c_{0}$ is the speed of sound, $S_{0}$ is a modified distance between the source and the observer, and $\mathscr{L}_{T I}$ is the turbulent inflow noise transfer function that connects the airfoil surface pressure fluctuation to the acoustic pressure at the far-field point. We neglect the second-order trailing-edge correction in the calculation of the transfer function, as given by Eq. (4) of Ref. [20], because it is small for the chords and frequencies considered in this study. Thus $\mathscr{L}_{T I}$ is obtained from Eq. (3) of Ref. [20].

Finally, $\Phi_{w w}$ is the two-dimensional energy spectrum, modeled here by a von Kármán spectrum for homogeneous and isotropic turbulence [10, 20]:

$$
\Phi_{w w}\left(K_{x}, K_{y}\right)=\frac{4}{9 \pi} \frac{\sigma_{u}^{2}}{K_{e}^{2}} \frac{\hat{K}_{x}^{2}+\hat{K}_{y}^{2}}{\left(1+\hat{K}_{x}^{2}+\hat{K}_{y}^{2}\right)^{7 / 3}},
$$

with $\sigma_{u}$ the standard deviation of turbulent velocity fluctuations, $K_{e}=1 / L_{\text {outer }}$ the wave number corresponding to the turbulence outer scale $L_{\text {outer }}$, and $\hat{K}=K / K_{e}$ the normalized turbulent wave number. $L_{\text {outer }}$ is related to the turbulence integral length scale $\Lambda$ by $\Lambda=L_{\text {outer }} / 1.339$ [10].
2.1.2. Airfoil thickness correction for turbulent inflow noise

Several authors including Roger and Moreau [20] and Devenport et al. [21] have shown that turbulent inflow noise strongly depends on the airfoil thickness. A thicker airfoil tends to reduce the turbulent inflow noise level. This effect is not taken into account in Amiet's original model. We propose here an empirical correction based on the data shown in Figure 6 of Reference [20]. The reduction level $S P L_{R}$ in $\mathrm{dB}$ is calculated by linear interpolation based on these data:

$$
S P L_{R}(d B)=\frac{9}{50} \frac{(e / c)}{(e / c)_{r e f}} \frac{f}{U} \frac{(\Lambda / c)_{r e f}}{(\Lambda / c)},
$$

where $e$ is the airfoil maximum thickness, and $\Lambda$ is the turbulent integral length scale. The subscript ref stands for the values of reference experimental data from a NACA 0012 airfoil, which are $(e / c)_{\text {ref }}=0.12$ and $(\Lambda / c) \approx 0.1$.

Note that $S P L_{R}$ provides a pure thickness correction but does not contain the effect of camber as well as nose radius (curvature). Another approach that could be considered in future studies would be to correct the incident turbulence spectrum based on rapid distortion theory, as proposed by Roger and Moreau [20].

\subsubsection{Model validation against wind tunnel experi- ments}

First, model predictions are compared to the measurements of Paterson and Amiet [22] in Figure 2 for a NACA 0012 of chord $23 \mathrm{~cm}$, with Mach numbers between 0.12 and 0.50 . The turbulence intensity is $4-5 \%$, and the longitudinal integral length scale is $3.0 \mathrm{~cm}$. The agreement between model and measurements is greatly improved when the thickness correction is included, which is expected since this set of data was used to obtain Eq. (3). The thickness correction is highest for high frequencies and low Mach numbers.

Second, the measurements of Devenport et al. [21] for a $\mathrm{S} 831$ airfoil of chord $91 \mathrm{~cm}$ are considered. This airfoil whose maximum thickness is $18 \%$ of the chord is typically used in wind turbine applications. The Mach number is 0.08 , the turbulence intensity $3.9 \%$, and the longitudinal integral length scale $82 \mathrm{~cm}$. In Figure 3, model predictions with and without thickness correction are compared to the measurements at angles of attack of $0^{\circ}$ and $2^{\circ}$. The thickness correction slightly improves the agreement between predictions and measurements, but is not sufficient to provide a satisfying agreement below $200 \mathrm{~Hz}$. This discrepancy can be attributed to AoA, curvature and camber effects. As noted by Devenport et al. [21], turbulent inflow noise is almost independent of AoA for symmetric airfoils such as NACA 0012, but is influenced by the airfoil geometry for the S831. This is clearly seen in 


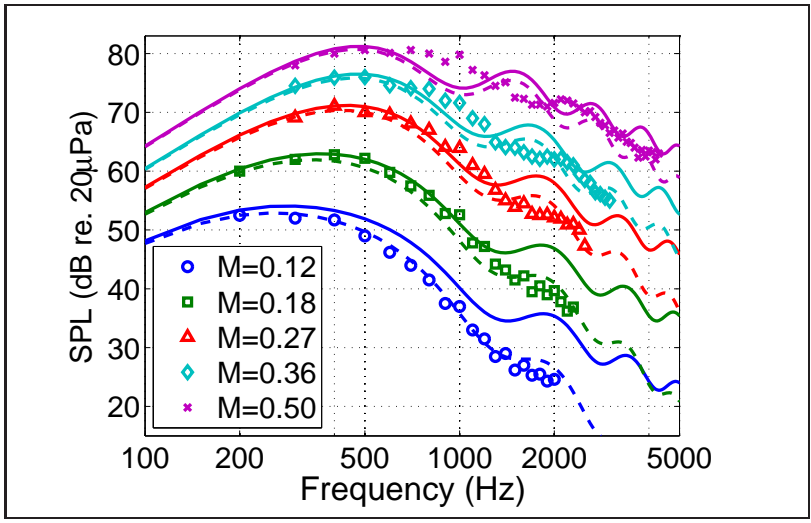

Figure 2: Turbulent inflow noise predictions with (dashed lines) and without (solid lines) thickness correction for a NACA 0012 airfoil. The symbols correspond to the measurements of Ref. [22] (color online).

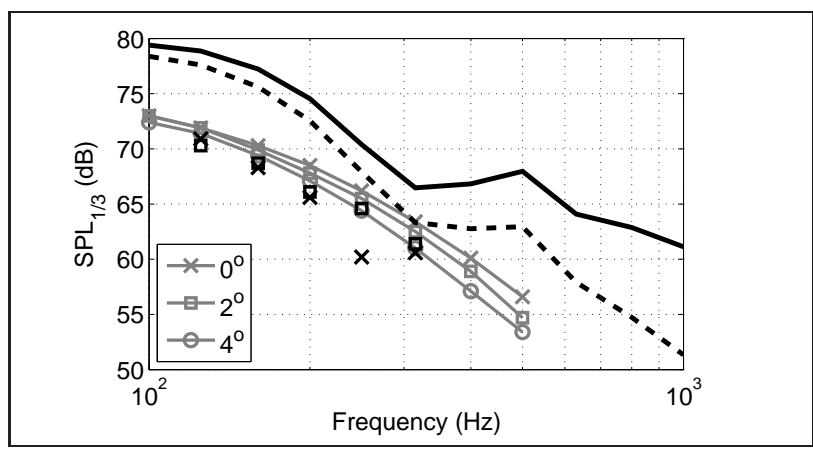

Figure 3: Turbulent inflow noise predictions with (black dashed lines) and without (black solid lines) thickness correction for a $\mathrm{S} 831$ airfoil. The results are compared to the measurements (black symbols) and predictions (gray lines) of Devenport et al. [21] for AoA of $0^{\circ}, 2^{\circ}$ and $4^{\circ}$.

the predictions of Devenport et al. [21] for AoA between $0^{\circ}$ and $4^{\circ}$, reproduced in Figure 3. These predictions are based on a panel method described in Ref. [23] that exactly accounts for the airfoil geometry. Figure 3 shows that Devenport et al. predictions correctly capture the thickness effect, and that the effect of AoA is significant, with a decrease of about $4 \mathrm{~dB}$ between $0^{\circ}$ and $4^{\circ}$. Even though Amiet's predictions tend to overestimate the noise levels in this case, one must keep in mind that the Mach number is very small in the Devenport et al. experiment, thus the discrepancy seen in Figure 3 can be considered as a worst case scenario for wind turbine blades.

\subsection{Trailing edge noise}

\subsubsection{Original model for a fixed plate}

Amiet's model originally proposed for turbulent inflow noise can be extended to trailing edge noise [11].
Assuming large aspect ratio, the PSD of trailing edge noise at far-field can be written as [11, 24]:

$$
\begin{aligned}
& S_{p p}\left(x_{R}, y_{R}, z_{R}, \omega\right)=\left(\frac{k c z_{R}}{4 \pi S_{0}^{2}}\right)^{2} \frac{L}{2} \\
& \Phi_{p p}(\omega) l_{y}\left(\omega, \frac{k y_{R}}{S_{0}}\right)\left|\mathscr{L}_{T E}\left(\frac{\omega}{U}, \frac{k y_{R}}{S_{0}}\right)\right|^{2}
\end{aligned}
$$

where $\Phi_{p p}$ is the wall pressure fluctuation spectrum, $l_{y}$ is the spanwise correlation length, estimated by the Corcos model, and $\mathscr{L}_{T E}$ is the transfer function for trailing edge noise. Roger and Moreau [20] showed that the second-order leading-edge correction has a significant contribution in the calculation of the transfer function only for $k c<1$. This condition is not encountered for the configurations studied here, so $\mathscr{L}_{T E}$ is simply obtained from Eq. (11) of Ref. [20]. As explained by Roger and Moreau [20], this expression includes the incident pressure jump correction proposed by Amiet [25].

\subsubsection{Wall pressure spectral models}

In Amiet's trailing edge noise model, one of the most important input parameters is the spectrum of wall pressure fluctuations. An accurate estimation of this spectrum can be done experimentally, or numerically with direct numerical simulation (DNS) or large eddy simulation (LES), but it remains very difficult in practice so a model must be used instead. As a completion of his model, Amiet proposed an empirical expression based on the scaling variable $\tilde{\omega}=\omega \delta^{*} / U_{e}$, with $\delta^{*}$ the boundary layer displacement thickness and $U_{e}$ the external velocity. More recently, Goody [15] proposed an improved wall pressure spectrum model that considers Reynolds effect. However, all these scaling models are based on zero pressure gradient (ZPG) flow conditions, that are only suitable for a flat plate at zero incidence. For a real airfoil, an adverse pressure gradient (APG) flow is usually present on the suction side near the trailing edge. Rozenberg et al. [16] proposed a model that takes into account the APG effect, and reduces to Goody's model for zero pressure gradient conditions. They suggested that normalized wall pressure spectrum can be presented as:

$$
\begin{aligned}
& \frac{\Phi_{p p}(\omega) U_{e}}{\tau_{\max }^{2} \delta^{*}}= \\
& \frac{\left[2.82 \Delta^{2}\left(6.13 \Delta^{-0.75}+F_{1}\right)^{A_{1}}\right]\left[4.2\left(\frac{\Pi}{\Delta}\right)+1\right] \tilde{\omega}^{2}}{\left[4.76 \tilde{\omega}^{0.75}+F_{1}\right]^{A_{1}}+\left[8.8 R_{T}^{-0.57} \tilde{\omega}\right]^{A_{2}}}
\end{aligned}
$$

where the main parameters of the model are [16]:

- the wake strength parameter $\Pi=0.8\left(\beta_{c}+0.5\right)^{3 / 4}$,

- the Clauser parameter $\beta_{c}=\frac{\theta}{\tau_{w}} \frac{d p}{d x}$ that compares pressure forces on the boundary layer to the wall shear forces,

- the ratio of boundary layer thickness to displacement thickness $\Delta=\delta / \delta^{*}$, 
- the ratio of the outer to inner boundary layer time scales $R_{T}=\frac{\delta}{U_{e}} \frac{\nu}{u_{\tau}^{2}}$

with $\theta$ the momentum thickness, $\tau_{w}$ the wall shear stress, $\tau_{\max }$ the maximum shear stress along the normal direction, and $\frac{d p}{d x}$ the pressure gradient. Finally, $A_{1}, A_{2}$ and $F_{1}$ are empirical coefficients given by:

$$
\begin{aligned}
& A_{1}=3.7+1.5 \beta_{c} \\
& A_{2}=\min \left(3,19 / \sqrt{R_{T}}\right)+7, \\
& F_{1}=4.76\left(\frac{1.4}{\Delta}\right)^{0.75}\left[0.375 A_{1}-1\right] .
\end{aligned}
$$

These parameters can be calculated using CFD tools. In this study, XFOIL version 6.96 is used to obtain $U_{e}$, $\delta^{*}, \theta$, the skin friction coefficient $C_{f}$ and the pressure coefficient $C_{p}$ at the trailing edge. The boundary layer thickness is obtained using the following relation [26]:

$$
\delta=\theta^{*}\left(3.15+\frac{1.72}{H_{k}-1}\right)+\delta^{*}
$$

where $H_{k}=\delta^{*} / \theta^{*}$ is the kinematic shape factor. We cannot estimate $\tau_{\max }$ directly from XFOIL, so we use the approximation $\tau_{\max } \approx \tau_{w}=\frac{1}{2} \rho U^{2} C_{f}$ that is valid while the boundary layer remains attached. Finally, the pressure gradient is obtained from $C_{p}$ between $99 \%$ and $100 \%$ of the chord.

\subsubsection{Model validation against wind tunnel experi- ments}

To validate Amiet's model and evaluate the effect of the adverse pressure gradient, results with Goody's model for ZPG and Rozenberg's model for APG are compared to experimental data from Brooks and Hodgson [27] for a NACA 0012 airfoil and from Kamruzzaman et al. [17] for a NACA 643-418 airfoil.

We consider first the surface pressure measurement of Brooks and Hodgson for a sensor located at $1.854 \mathrm{~cm}$ from the trailing edge. The airfoil is a NACA 0012 of chord $61 \mathrm{~cm}$ at zero incidence, and the inflow velocity is $69.5 \mathrm{~m} / \mathrm{s}$. Figure 4(a) compares ZPG and APG wall pressure spectra with the measured spectrum. The APG model is seen to increase the spectral level below $5 \mathrm{kHz}$ which provides a better agreement compared to the measurements. In Figure 4(b), sound pressure level (SPL) predictions are compared to experimental values. Predicted results are closer to the measurements using the APG model.

Kamruzzaman et al. [17] have performed surface pressure measurements on an asymmetric NACA $64_{3}-$ 418 airfoil of chord $60 \mathrm{~cm}$ on both pressure and suction sides. The inflow velocity is $62 \mathrm{~m} / \mathrm{s}$ and the AoA is $0^{\circ}$. The boundary layer displacement thickness $\delta^{*}$ and momentum thickness $\theta^{*}$ calculated by XFOIL are compared to measured values in Table I. Associated wall pressure spectra are plotted in Figure 5(a). On the suction side, the predictions are much closer to the measurements using the APG model compared to the

\begin{tabular}{|c|c|c|}
\hline & $\delta^{*}(\mathrm{~mm})$ & $\theta^{*}(\mathrm{~mm})$ \\
\hline Experiment & 6.76 & 2.99 \\
\hline XFOIL & 5.97 & 3.07 \\
\hline
\end{tabular}

Table I: Boundary layer parameters calculated by XFOIL and measured by Kamruzzaman et al. [17] on the suction side at the trailing edge for an AoA of $0^{\circ}$.

ZPG model, although the levels are still lower than the measured ones. On the pressure side, only the ZPG model is used because pressure gradients are small. Figure 5(b) compares the SPL spectrum predictions to the measurements. Using the APG model on the suction side and the ZPG model on the pressure side, a better agreement is found although the predictions still underestimate the measured values.

As a conclusion, it is clear that the adverse pressure gradient has an important effect on the SPL prediction, however its modeling is still an open issue in the aeroacoustics community. Some recent studies have shown that turbulence anisotropy effects need to be included to improve the model accuracy, using for instance the TNO-Blake model [17, 18].

\section{Application on a full size wind tur- bine with constant wind profiles}

In this section, we consider a constant wind profile (no wind shear) and no atmospheric turbulence. Thus only trailing edge noise is considered in the SPL predictions.

\subsection{Model adaption to a rotating blade with spanwise-varying flow conditions}

Amiet's model was originally developed for a fixed plate. A simple method to account for the blade rotating motion consists in approximating it by a series of translations from discrete angular positions, as explained by Schlinker and Amiet [28]. This approximation has recently been revisited by Blandeau and Joseph [29] and by Sinayoko et al. [30] by comparison with analytical models that treat the rotation effects exactly. They concluded that the approximation is valid over a wide range of frequencies for wind turbine applications. Using Blandeau and Joseph expressions [29], frequency limits can be obtained as a function of distance from an observer to the wind turbine as well as Reynolds number encountered by each blade section. The low frequency limits are about $15 \mathrm{~Hz}$ and $120 \mathrm{~Hz}$ for an observer located respectively $100 \mathrm{~m}$ and $1000 \mathrm{~m}$ away from the wind turbine. The upper frequency limit increases with Reynolds number. In our study, the average Reynolds number is around $4 \times 10^{6}$, which leads to an upper frequency limit from $1 \mathrm{kHz}$ to $5 \mathrm{kHz}$ from the root to the tip. Since most of the wind turbine noise is produced by the outer part of the 


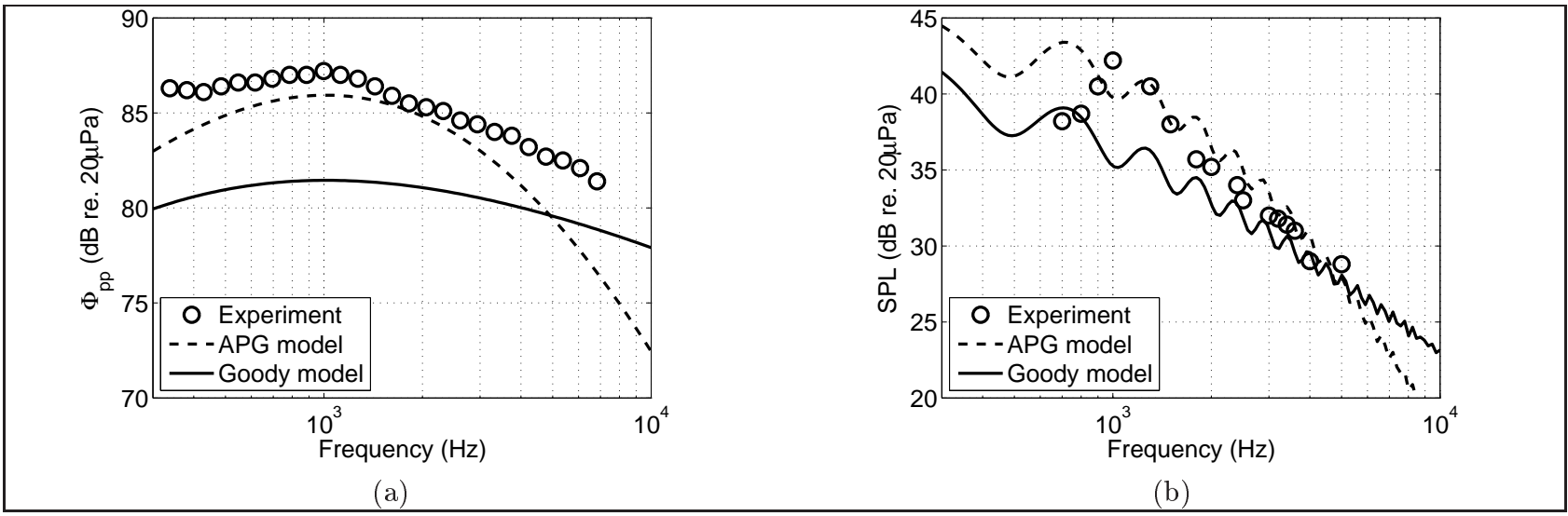

Figure 4: (a) Wall pressure spectra and (b) far-field SPL predicted by APG and ZPG models and measured by Brooks and Hodgson [27] for a NACA 0012 airfoil.

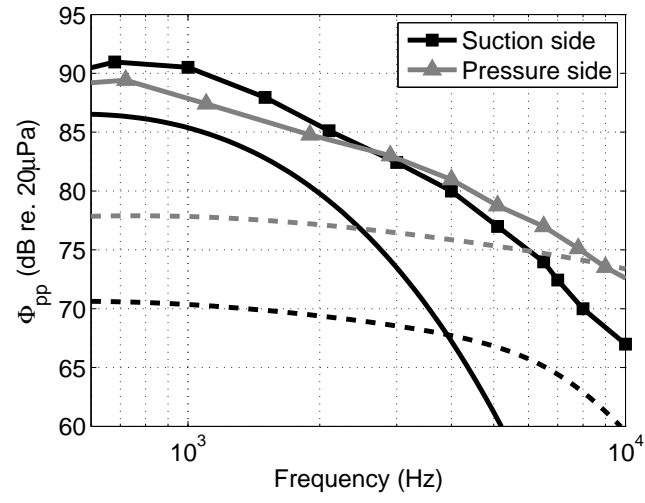

(a)

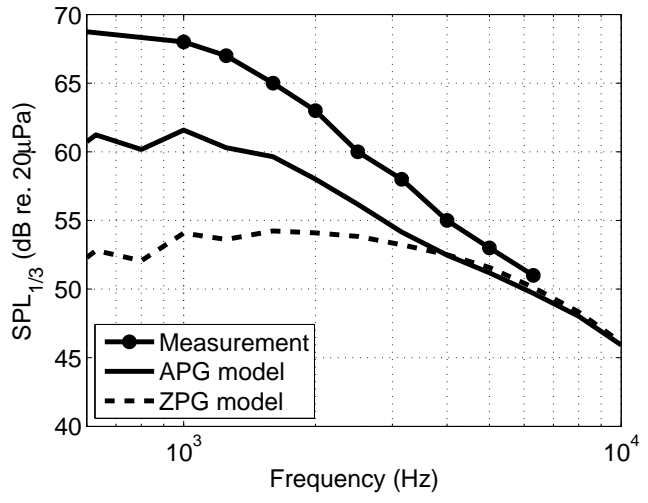

(b)

Figure 5: (a) Wall pressure spectra on the suction side (black lines) and on the pressure side (gray lines) measured by Kamruzzaman et al. [17] (symbols) and predicted by APG (solid lines) and ZPG models (dashed lines) models. (b) Third octave band spectra of far-field SPL using APG or ZPG model on the suction side and ZPG model on the pressure side.

blade, as observed by Oerlemans et al. [31], results are calculated up to $5 \mathrm{kHz}$ in the following.

The Doppler factor relates the observer frequency $\omega$ to the emission frequency $\omega_{e}$ at the source [28, 30]. As shown by Schlinker and Amiet [28] and Sinayoko et al. [30], the instantaneous PSD at the observer for an azimuthal blade position $\gamma$ is $S_{p p}\left(\mathbf{x}_{\mathbf{0}}, \omega, \gamma\right)=\left(\omega_{e} / \omega\right) S_{p p}^{\prime}\left(\mathbf{x}, \omega_{e}, \gamma\right)$, where $\mathbf{x}_{\mathbf{0}}$ and $\mathbf{x}$ correspond respectively to the observer coordinates in the hub and blade coordinate systems, and $S_{p p}^{\prime}\left(\mathbf{x}, \omega_{e}, \gamma\right)$ is given by Equation (4) (or Equation (1) when turbulent inflow noise is considered) for a fixed blade. They also derived an expression for the az- imuthally averaged spectrum:

$$
\begin{aligned}
S_{p p}\left(\mathbf{x}_{\mathbf{0}}, \omega\right) & =\frac{1}{2 \pi} \int_{0}^{2 \pi} \frac{\omega_{e}}{\omega} S_{p p}\left(\mathbf{x}_{\mathbf{0}}, \omega, \gamma\right) d \gamma \\
& =\frac{1}{2 \pi} \int_{0}^{2 \pi}\left(\frac{\omega_{e}}{\omega}\right)^{2} S_{p p}^{\prime}\left(\mathbf{x}, \omega_{e}, \gamma\right) d \gamma
\end{aligned}
$$

Another issue related to blade rotation is that the flow is not uniform along the span, with incoming velocity strongly increasing from root to tip. To treat these spanwise-varying conditions, it is common to cut the blade into short segments or strips while assuming the segments are independent, which means the segment span must be greater than the spanwise turbulence correlation length. As a result, the overall noise radiated by the blade is the logarithmic sum of the contributions from all blade segments. 

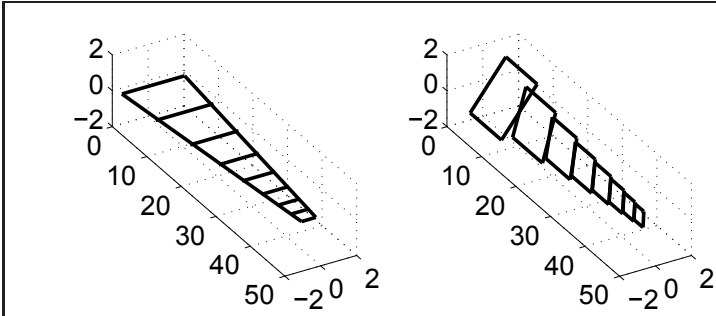

Figure 6: Geometry of the $45 \mathrm{~m}$-blade cut into 8 segments without twist (left) and with twist (right). All dimensions are in meters.

\begin{tabular}{|c|c|c|}
\hline & $\begin{array}{c}\text { Wind speed at } \\
\text { hub height }(\mathrm{m} / \mathrm{s})\end{array}$ & $\begin{array}{c}\text { Rotor speed } \\
(\mathrm{rpm})\end{array}$ \\
\hline case 1 & 6 & 13 \\
case 2 & 8 & 14 \\
\hline
\end{tabular}

Table II: Mean parameters for the two experimental test-cases from Reference [32].

\subsection{Configurations}

The wind turbine under study is a $2.3 \mathrm{MW}$ Siemens SWT 2.3-93 with a tower height (ground to hub) of $80 \mathrm{~m}$, and three $45 \mathrm{~B}$ blades of length $45 \mathrm{~m}$ that have controllable pitch angle. The chord length is $3.5 \mathrm{~m}$ at the root of the blade and $0.8 \mathrm{~m}$ at the tip, and we assume a linear variation in-between as shown in Figure 6 . These data in addition to the sound power level measurements are found in Reference [32] for the two cases summarized in Table II.

A NACA 63-415 airfoil is chosen for the blade profile, because it is a commonly used airfoil in modern wind turbines, and it is visually similar to B45 blades [33]. To choose the number of blade segments, we decide in this study to keep a constant aspect ratio of 3 , so that the large aspect ratio approximation of Amiet's model is satisfied. This led us to cut each blade into 8 segments, as shown in Figure 6. The span is always larger than $0.5 \mathrm{~m}$, the largest spanwise correlation length according to Corcos model. Finally, the blade twist is chosen so that the AoA is $4^{\circ}$ with a constant wind profile for all segments, which is the angle where the maximum lift drag ratio is found for a Reynolds number of $4 \times 10^{6}$. A schematics of the twisted blade as it is modeled in the calculations is represented in Figure 6.

\subsection{Sound power calculation and comparison with measurements}

Assuming free field conditions, the sound power level $S W L$ is obtained by $S W L=S P L+10 \log _{10}\left(4 \pi R^{2}\right)$, with $R$ the distance from the rotor to the observer. The SWL predictions are compared to the measurements in Figures 7 and 8 for the two cases described in

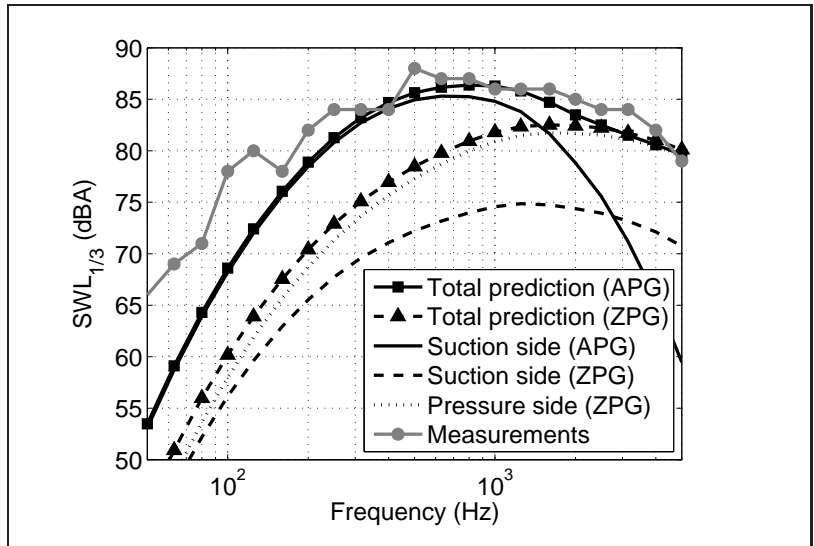

Figure 7: Third octave band spectra of sound power level for case $1(U=6 \mathrm{~m} / \mathrm{s})$ considering APG or ZPG models of trailing edge noise.

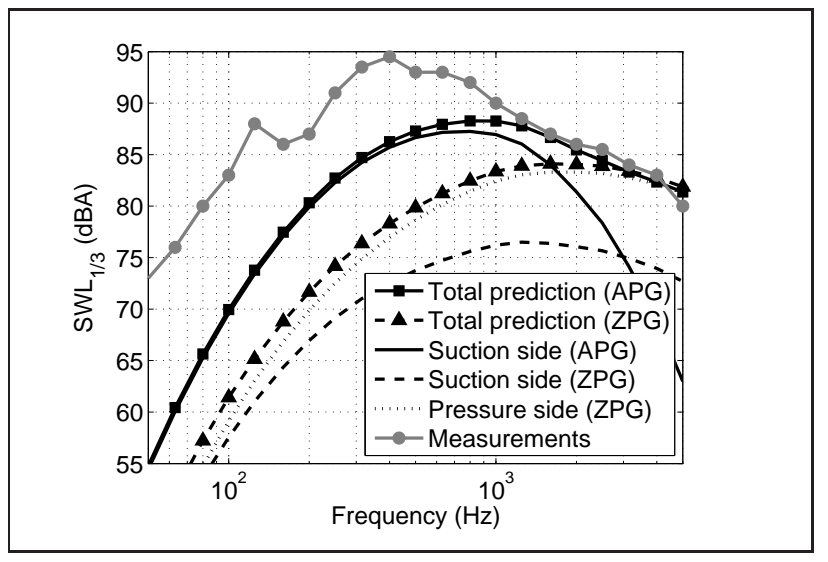

Figure 8: Third octave band spectra of sound power level for case $2(U=8 \mathrm{~m} / \mathrm{s})$ considering APG or ZPG models of trailing edge noise.

Table II. The observer is located on the ground $100 \mathrm{~m}$ downwind, and the spectra are azimuthally averaged as given by Equation (10). Using the APG model on the suction side, the predictions agree well at high frequencies, above $200 \mathrm{~Hz}$ for case 1 and $1000 \mathrm{~Hz}$ for case 2 . For both cases, trailing edge noise is dominated by the suction side contribution at lower frequencies, and by the pressure side contribution at higher frequencies. Using the ZPG model on the suction side, the predictions are up to $10 \mathrm{~dB}$ lower compared to the APG model predictions, and are lower than measurements over the whole frequency range. At low frequencies, both model predictions underestimate the measurements, which can be attributed to the fact that other noise mechanisms dominate in this frequency range, as will be seen in Section 4.4 .

\subsection{Directivity and amplitude modulation}

The horizontal directivity of overall SPL is plotted in Figure 9(a) for cases 1 and 2. The maximum levels are 


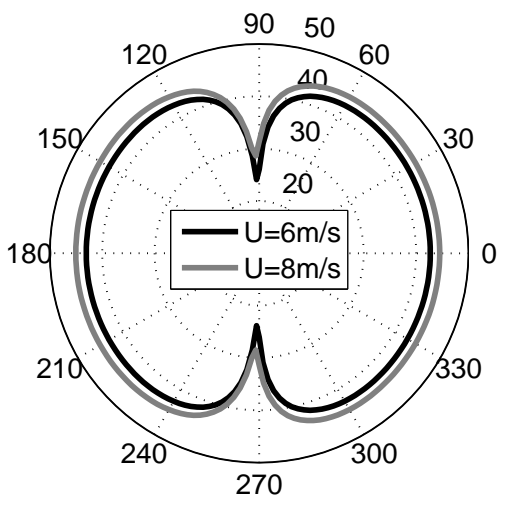

(a)

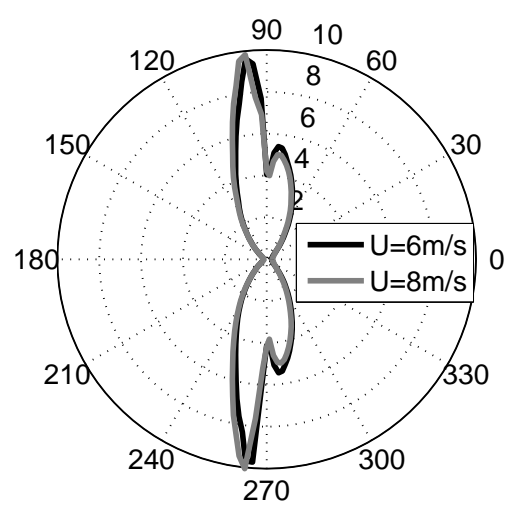

(b)

Figure 9: Horizontal directivity of (a) overall SPL and (b) amplitude modulation strength, with the wind coming from the left.

obtained upwind and downwind, while the minimum levels are found crosswind, which is in agreement with typical field measurements close to a wind turbine [2]. This shape can be explained by the directivity of trailing edge noise, coming from the assumption of dipole distribution in Amiet's theory. This directivity is determined by the orientation of the blade.

Amplitude modulation is caused by the rotation of the blades, and has a frequency of $1 / 3$ the blade rotating frequency for a 3-bladed wind turbine. Subtracting the mean SPL from the SPL at each blade azimuthal position $\gamma$, we can visualize AM in Figure 10 for observers in downwind and crosswind direction. The AM is almost identical for cases 1 and 2 . The variations are small in the downwind direction, and much more important in the crosswind directions. We define the AM strength as the difference between minimum and maximum values of SPL over blade azimuthal position $\gamma$. The AM strength is approximately $4 \mathrm{~dB}(\mathrm{~A})$ crosswind and less than $0.3 \mathrm{~dB}(\mathrm{~A})$ downwind. Figure $9(\mathrm{~b})$ shows the directivity of AM strength for cases 1 and 2. Large values of $\mathrm{AM}$ strength, of up to $10 \mathrm{~dB}(\mathrm{~A})$, are found in the vicinity of the crosswind directions, where the minimum overall SPL values are found according to Figure 9(a). These predictions are in good qualitative agreement with field measurements [2], and can be explained by rotation and directivity effects.

\section{Influence of atmospheric turbu- lence and wind shear}

\subsection{Monin-Obukhov similarity theory}

Monin-Obukhov Similarity Theory (MOST) is considered to study the influence of atmospheric turbulence and wind shear on wind turbine noise. This theory applies to the atmospheric surface layer, where surface fluxes are relatively constant, and is valid over a flat

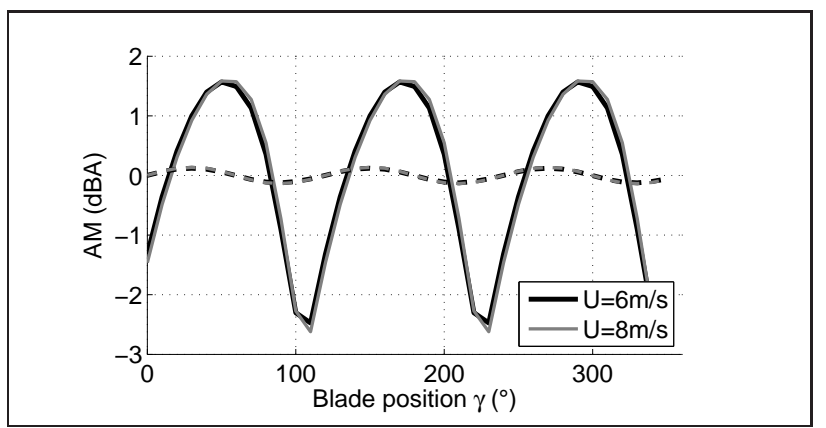

Figure 10: Amplitude modulation in downwind (dashed lines) and cross-wind (solid lines) directions for cases 1 and 2. The observer is $100 \mathrm{~m}$ away from the wind turbine, and AM is obtained by subtracting from the mean $\mathrm{SPL}$ from $\operatorname{SPL}(\gamma)$.

and homogeneous ground $[34,35,36]$. The main parameters of the model are the friction velocity $u_{*}$ and the sensible heat flux $H$, or equivalently the temperature scale $T_{*}$. The stability of the atmosphere is then described by the Obukhov length $L_{*}$ given by [34, 36]:

$$
L_{*}=\bar{T} u_{*}^{2} /\left(\kappa g T_{*}\right)=-\frac{\rho_{0} C_{p} \bar{T} u_{*}^{3}}{\kappa g H},
$$

with $\bar{T}$ the potential temperature, $\kappa=0.41$ the von Kármán constant, $g$ the gravity acceleration, and $C_{p}$ the specific heat of dry air. The atmosphere is unstable when $L_{*}<0(H>0)$ and stable when $L_{*}>0$ $(H<0)$. When the shear production of turbulence is much larger than the buoyant production, the atmosphere is called neutral and $1 / L_{*} \approx 0(H \approx 0)$.

The mean velocity profile as a function of height $z$ can then be obtained using similarity relations [37, 34]:

$$
U(z)=\frac{u_{*}}{\kappa}\left[\ln \left(\frac{z}{z_{0}}\right)-\psi_{u}\right],
$$




\begin{tabular}{|c|c|c|}
\hline \multicolumn{3}{|c|}{ Case 1: $U(80 \mathrm{~m})=6 \mathrm{~m} / \mathrm{s}$} \\
\hline$H\left(\mathrm{~W} / \mathrm{m}^{2}\right)$ & $u_{*}(\mathrm{~m} / \mathrm{s})$ & $L_{*}(\mathrm{~m})$ \\
\hline-10 & 0.29 & 235 \\
\hline 0 & 0.37 & Inf \\
\hline 40 & 0.42 & -168 \\
\hline \hline \multicolumn{3}{|c|}{ Case $2: U(80 \mathrm{~m})=8 \mathrm{~m} / \mathrm{s}$} \\
\hline$H\left(\mathrm{~W} / \mathrm{m}^{2}\right)$ & $u_{*}(\mathrm{~m} / \mathrm{s})$ & $L_{*}(\mathrm{~m})$ \\
\hline-25 & 0.38 & 200 \\
\hline-10 & 0.46 & 905 \\
\hline 0 & 0.49 & Inf \\
\hline 40 & 0.53 & -348 \\
\hline 200 & 0.58 & -92 \\
\hline
\end{tabular}

Table III: MOST parameters used in the study for cases 1 and 2 .

where $z_{0}$ is the surface roughness length and the function $\psi_{u}$ depends on the stability of the atmosphere. In neutral conditions, $\psi_{u}=0$ and the classical logarithmic profile is recovered. These velocity profiles are sometimes called Businger-Dyer profiles, and we use in this study a slightly modified version of these profiles detailed in Appendix A. Using MOST, it is also possible to predict turbulence parameters that vary with height to represent the inhomogeneity of the atmospheric boundary layers. The von Kármán spectrum of Equation (2) is used, but with heightdependent standard deviation of turbulent velocity fluctuations $\sigma_{u}$ and integral length scale $\Lambda$ that are described in Appendix A.

Since detailed parameters concerning the atmospheric conditions during the wind turbine noise measurements are not mentioned in Reference [32], we choose realistic parameters found in the literature. The heat flux $H$ typically varies over the range $-50 \mathrm{~W} / \mathrm{m}^{2}$ to $600 \mathrm{~W} / \mathrm{m}^{2}$ during a diurnal cycle [34]. Following Ostashev and Wilson [38], we select a value of $200 \mathrm{~W} / \mathrm{m}^{2}$ for mostly sunny conditions, and $40 \mathrm{~W} / \mathrm{m}^{2}$ for mostly cloudy conditions. For stable conditions, typically occurring at night, values of $-10 \mathrm{~W} / \mathrm{m}^{2}$ and $-25 \mathrm{~W} / \mathrm{m}^{2}$ are chosen for $H$. Then we deduce the friction velocity from Equation (12) so that the mean velocity at hub height is $6 \mathrm{~m} / \mathrm{s}$ for case 1 or $8 \mathrm{~m} / \mathrm{s}$ for case 2 , using $z_{0}=0.1 \mathrm{~m}$. The results are summarized in Table III. Results for $H=-25 \mathrm{~W} / \mathrm{m}^{2}$ and $200 \mathrm{~W} / \mathrm{m}^{2}$ are not shown for case 1 because they yield $\left|L_{*}\right|<50 \mathrm{~m}$, and it is generally admitted that MOST is only valid for $\left|z / L_{*}\right|<1-2[35]$. Let us note that for $H=200 \mathrm{~W} / \mathrm{m}^{2}$ and $U(80 \mathrm{~m})=8 \mathrm{~m} / \mathrm{s}$, the validity of MOST might be questionable for the highest part of the rotor.

The different possible wind profiles are plotted in Figure 11 for case 2 . The wind shear is clearly stronger in stable conditions compared to neutral or unstable conditions. The wind speed increases from 6.2 to $9.5 \mathrm{~m} / \mathrm{s}$ between the bottom and top parts of the rotor for $H=-25 \mathrm{~W} / \mathrm{m}^{2}$, while it remains close to $8 \mathrm{~m} / \mathrm{s}$

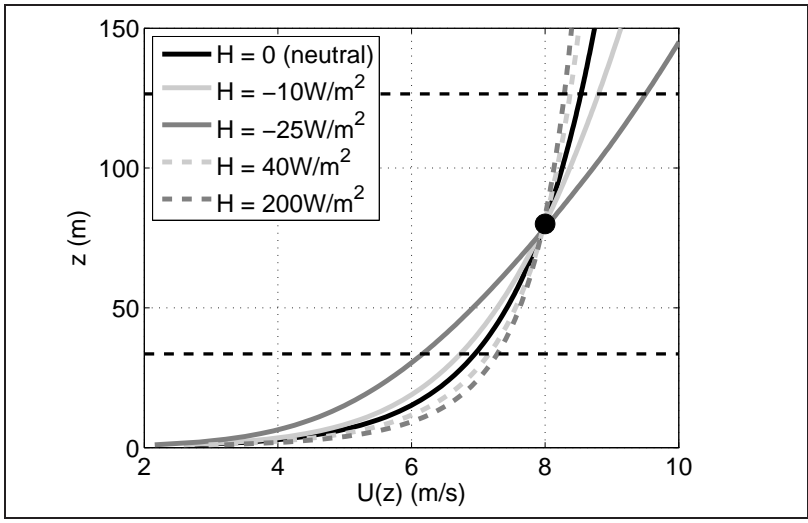

Figure 11: Mean wind profiles $U(z)$ for the atmospheric conditions described in Table III for case 2 $(U(80 \mathrm{~m})=8 \mathrm{~m} / \mathrm{s})$. The minimum and maximum rotor heights are shown using black dashed lines.

for unstable conditions. Similar results are obtained for case 1 so they are not plotted here. The turbulence parameters $\sigma_{u}$ and $\Lambda$ are plotted for case 2 in Figure 12. Using expressions given by Cheinet [36], $\sigma_{u}$ is independent of height in neutral and unstable conditions, while it increases with height in stable conditions. The integral length scale always increases with height, but in a much quicker way in stable atmospheres. The turbulence level associated with the von Kármán spectrum will thus be a combination of these two effects, as this level increases with increasing $\sigma_{u}$ and decreases with increasing $\Lambda$.

\subsection{Effect of wind shear on wind turbine trailing edge noise}

The noise radiated by a wind turbine depends on wind shear, mostly because an increase in wind speed causes an increase of the AoA seen by a blade segment. As an example, the variation of AoA over the rotor plane due to wind shear is plotted in Figure 13 for case 2 with $H=-25 \mathrm{~W} / \mathrm{m}^{2}$. The maximum AoA variation over one rotation is approximately $\pm 1.5^{\circ}$ for the tip segment. As a result, the turbulent boundary layer parameters vary with blade azimuthal position $\gamma$. For instance, Figure 14 shows the variation of the displacement thickness $\delta_{s}^{*}$ on the suction side for the different wind profiles corresponding to case 2 . The boundary layer thickness of the tip segment decreases from $\gamma=0$, where the blade is pointing up to $\gamma=180^{\circ}$, where the blade is pointing down. This decrease is most significant for the stable atmosphere with $H=-25 \mathrm{~W} / \mathrm{m}^{2}$. These changes in boundary layer parameters cause a significant change in the wall pressure spectra plotted in Figure 15 as a function of $\gamma$. The spectral peak shifts to higher frequency when the blade goes from top to down positions, corresponding to a decrease of AoA from $5.2^{\circ}$ to $2.5^{\circ}$. These spectral variations due to wind shear are in good 


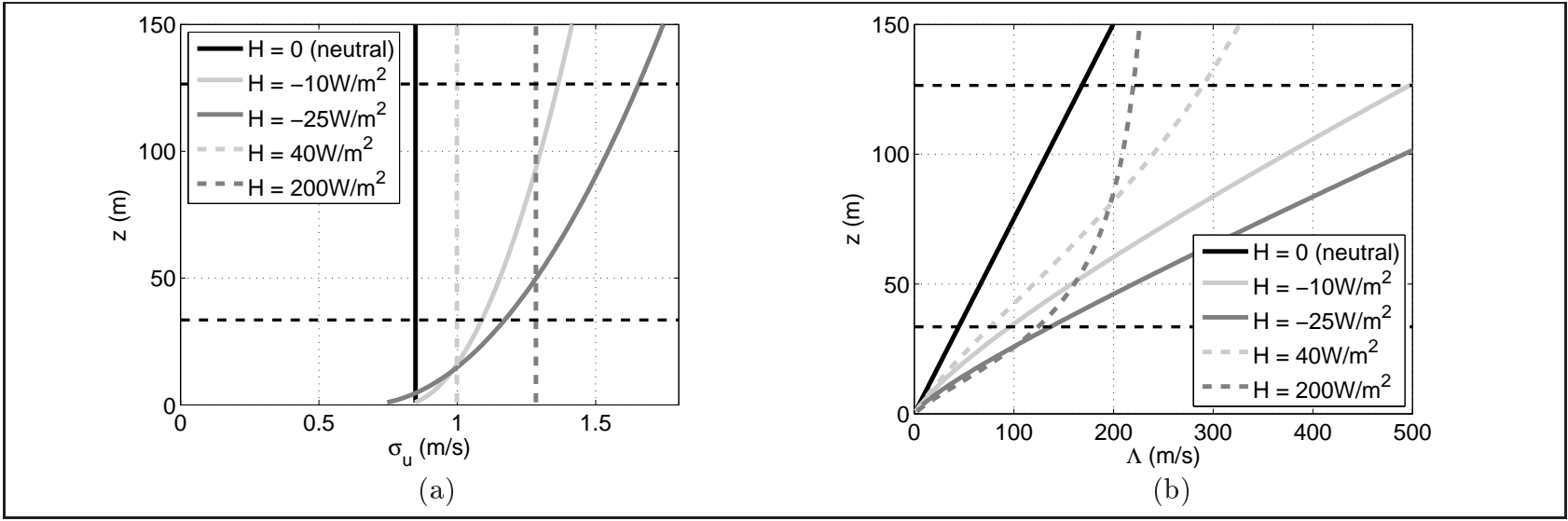

Figure 12: (a) Standard deviation of turbulent velocity fluctuations $\sigma_{u}$ and (b) integral length scale $\Lambda$ for $U(80 \mathrm{~m})=8 \mathrm{~m} / \mathrm{s}$. The minimum and maximum rotor heights are shown using black dashed lines.

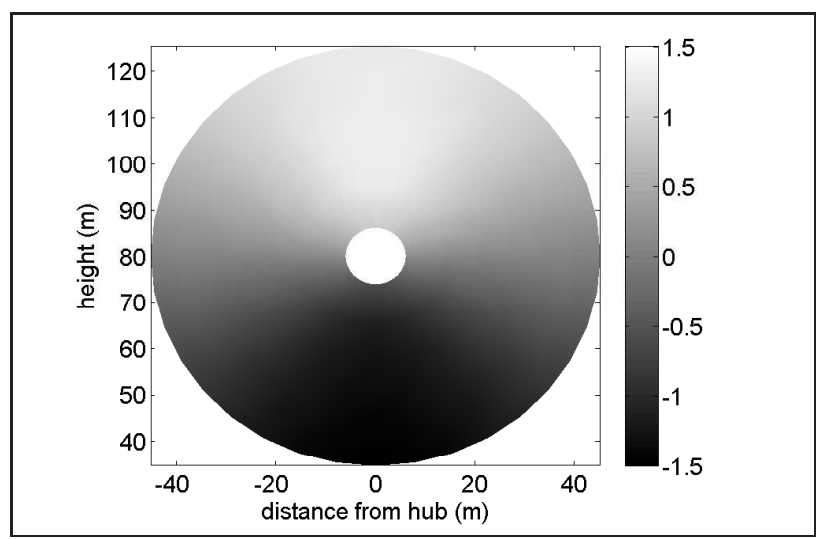

Figure 13: AoA variation in degrees due to wind shear for $U(80 \mathrm{~m})=8 \mathrm{~m} / \mathrm{s}$ and $H=-25 \mathrm{~W} / \mathrm{m}^{2}$.

agreement with surface pressure measurements performed in the framework of the DANAERO project for a similar size wind turbine [5, Figure 25].

This significant effect of wind shear on the emission side is much less pronounced on the receiver side, as can be seen in the sound power level spectra of Figure 16 calculated at a receiver $100 \mathrm{~m}$ downwind. On the suction side, a $S W L$ increase is observed at high frequencies, of approximately $1 \mathrm{~dB}(\mathrm{~A})$ at $2 \mathrm{kHz}$ and $3 \mathrm{~dB}(\mathrm{~A})$ at $4 \mathrm{kHz}$. However, this increase is not observed on the pressure side, and since pressure side levels dominate above $2 \mathrm{kHz}$, the maximum increase due to wind shear is only $0.4 \mathrm{~dB}(\mathrm{~A})$ at $2 \mathrm{kHz}$ on the total trailing edge noise spectrum. The fact that level variations due to wind shear are less pronounced on the receiver side (Figure 16) compared to the emission side (Figure 15) may be explained by the fact that the 3 blades and all the blade segments are considered to calculate the azimuthally-averaged spectra of Figure 16, thus variations due to wind shear tend to be averaged out. We must keep in mind that stronger

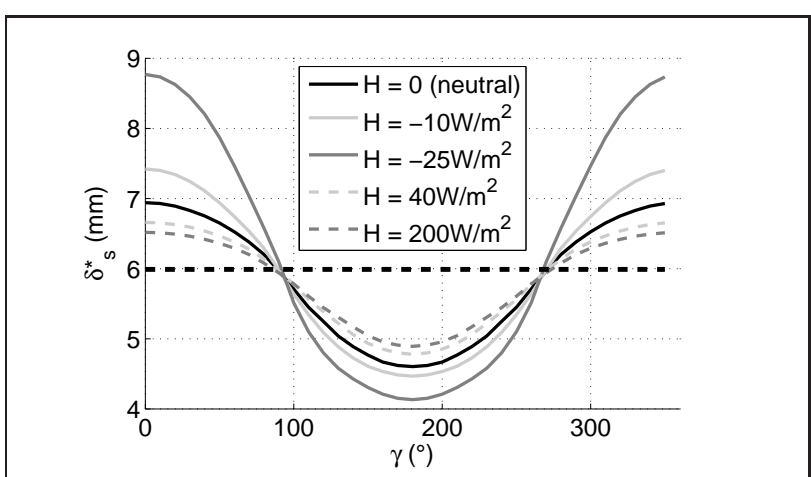

Figure 14: Variation of displacement thickness $\delta_{s}^{*}$ on the suction side as a function of blade azimuthal position $\gamma$ for the tip segment and for case 2. The thick dashed line corresponds to the reference value with a constant wind of $8 \mathrm{~m} / \mathrm{s}$.

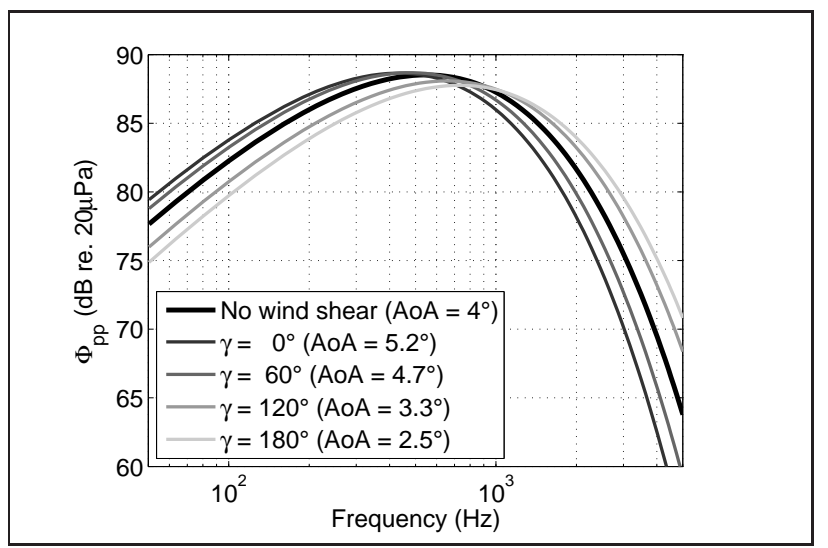

Figure 15: Wall pressure spectra $\Phi_{p p}$ on the suction side for different blade azimuthal position $\gamma$ for the tip segment, considering case 2 with $H=-25 \mathrm{~W} / \mathrm{m}^{2}$. 


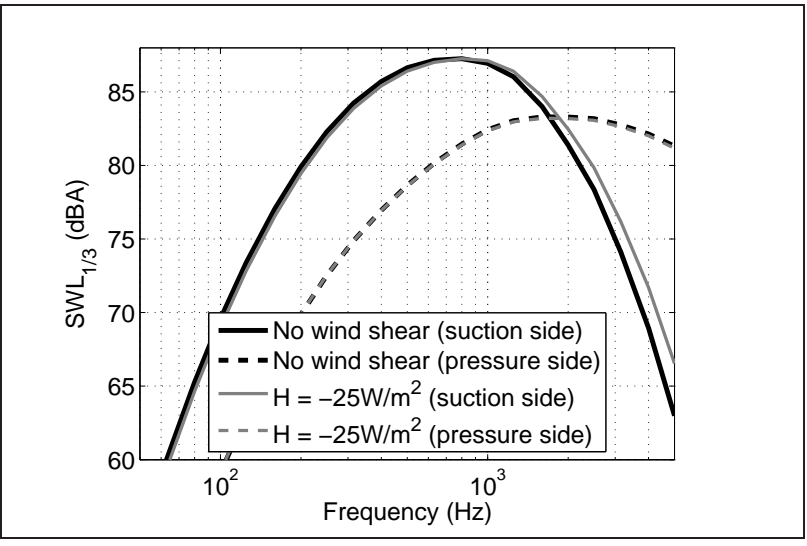

Figure 16: Third octave band spectrum of $S W L$ for trailing edge noise on pressure and suction side for case 2 with no wind shear and with $H=-25 \mathrm{~W} / \mathrm{m}^{2}$.

wind shear than those predicted by MOST usually exist in reality, because in practice the terrain might not be flat and homogeneous (topography effects), and because other sources of inhomogeneities such as largescale turbulence or wakes of other turbines might be present $[4,3,5]$.

\subsection{Effect of atmospheric turbulence on wind turbine turbulent inflow noise}

Amiet's model for turbulent inflow noise directly depends on the turbulence spectrum $\Phi_{w w}$, as seen in Equation (1). It is modeled using a von Kármán spectrum with turbulence parameters $\sigma_{u}$ and $\Lambda$ that depend on height or equivalently on the blade azimuthal position $\gamma$. Figure 17 shows how the turbulence spectrum varies with $\gamma$ for the tip segment at $100 \mathrm{~Hz}$. The maximum spectral levels are found for the unstable atmosphere with $H=200 \mathrm{~W} / \mathrm{m}^{2}$ during the whole rotation, which can be explained by the relatively high value of $\sigma_{u}$ and low value of the turbulent length scale $\Lambda$ associated with this case (see Figure 12). The same trends are observed for other frequencies.

Figure 18 shows the azimuthally-averaged $S W L$ spectra calculated at a receiver $100 \mathrm{~m}$ downwind associated with the same atmospheric conditions. As it could be foreseen from Figure 17, the maximum levels are obtained for $H=200 \mathrm{~W} / \mathrm{m}^{2}$ and the minimum levels for $H=0$ and $H=40 \mathrm{~W} / \mathrm{m}^{2}$. The differences are significant - up to $2 \mathrm{~dB}(\mathrm{~A})$ - between the different atmospheric conditions. As already mentioned previously for trailing edge noise, we must keep in mind that stronger turbulent variations than those predicted by MOST may exist in reality, especially when a wind turbine happens to be in the wake of another turbine $[3,5]$. To conclude this part, let us note that the leading edge thickness correction presented in Section 2.1.2 has a negligible effect on the final results. Indeed, integral length scales $\Lambda$ are much larger than the blade chord $c$, thus $\Lambda / c$ in Equation 3 is

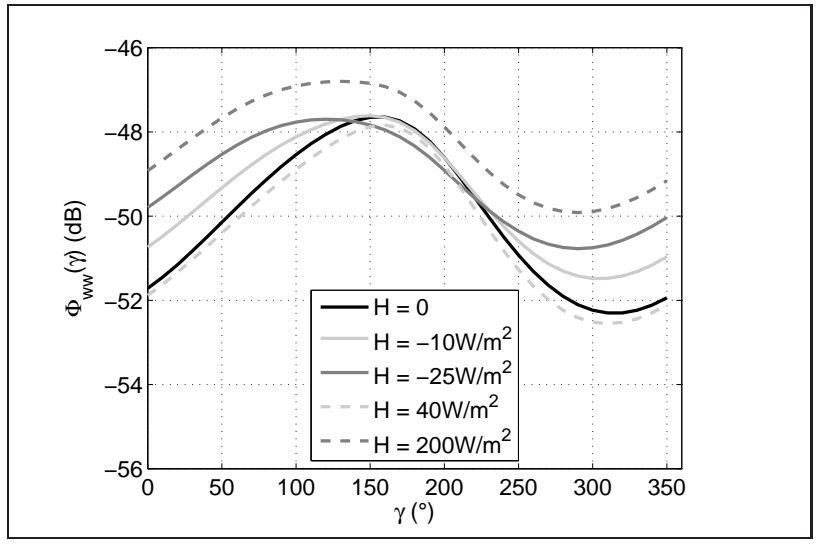

Figure 17: Variations of turbulence spectrum $\Phi_{w w}$ as a function of blade azimuthal position $\gamma$ for the tip segment at $100 \mathrm{~Hz}$ and for the various atmospheric conditions corresponding to case 2 .

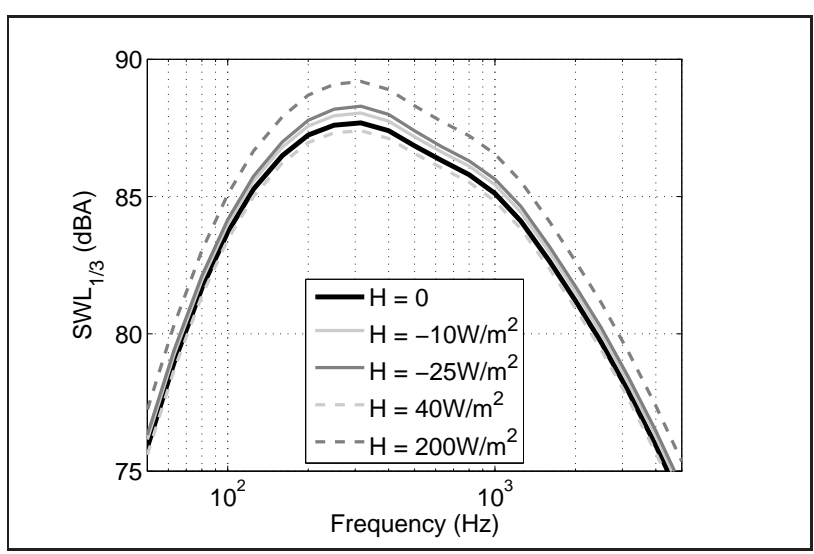

Figure 18: Third octave band spectrum of $S W L$ for turbulent inflow noise and for the various atmospheric conditions corresponding to case 2 .

large and $S P L_{R}$ is small, with a maximum reduction of $0.3 \mathrm{~dB}$ obtained for the root segment.

\subsection{Combined effects of wind shear and at- mospheric turbulence}

\subsubsection{Sound power level predictions}

The total $S W L$ spectra including both trailing edge noise and turbulent inflow noise are compared to measurements of Reference [32] in Figures 19 and 20 for cases 1 and 2 . We consider here a neutral atmosphere $(H=0)$, which means the turbulent inflow noise levels are relatively low according to Figure 18. It appears that turbulent inflow noise is dominant at low frequencies, up to 300 to $500 \mathrm{~Hz}$, while trailing edge noise is dominant at higher frequencies. The agreement between predictions and measurements is now quite satisfactory along the whole frequency band. For case 1 , predictions slightly overestimate the measurements at low frequency, which might indicate that the 


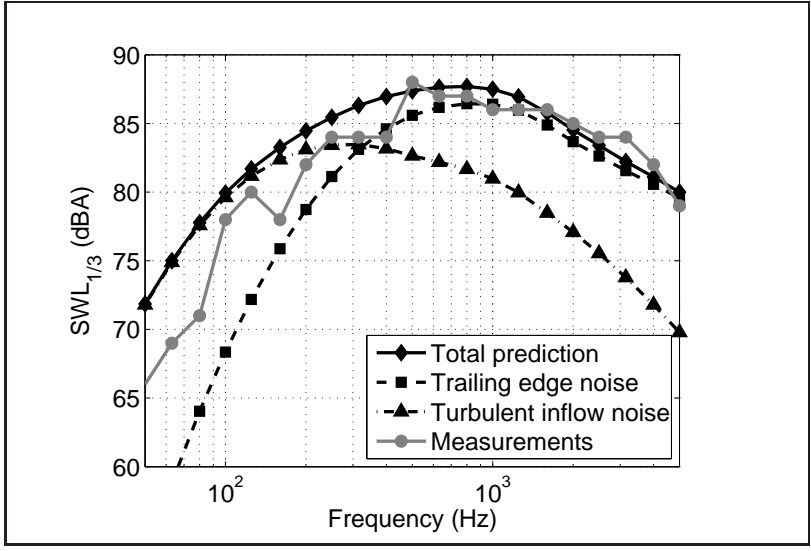

Figure 19: Third octave band spectrum of $S W L$ for trailing edge noise and turbulent inflow noise for case 1 and $H=0$ (neutral atmosphere).

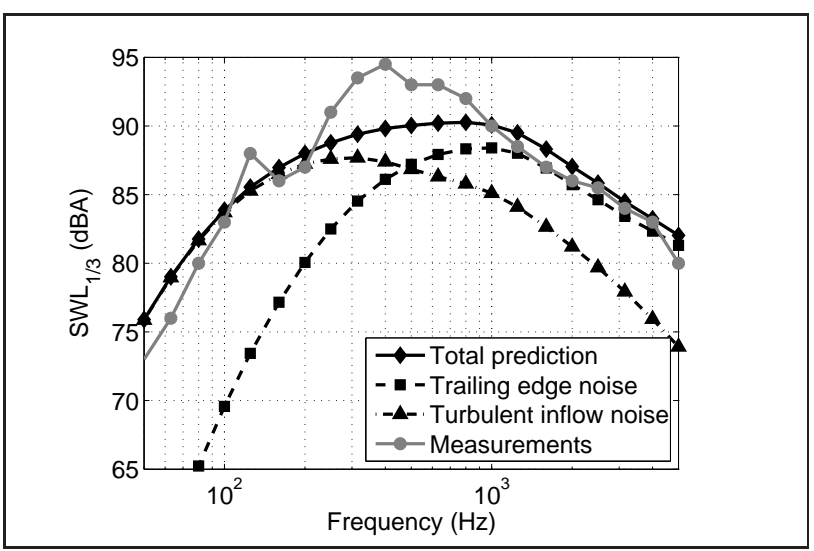

Figure 20: Third octave band spectrum of $S W L$ for trailing edge noise and turbulent inflow noise for case 2 and $H=0$ (neutral atmosphere).

turbulent inflow noise needs some improvements at these frequencies and/or that the atmospheric turbulence parameters are not well modeled. For case 2, the experimental spectral peak around $400 \mathrm{~Hz}$ is not captured by the model, which may be due to the absence of other noise sources in the predictions such as separation/stall noise.

\subsubsection{Directivity and amplitude modulation}

The horizontal directivities of overall SPL and of AM strength are plotted in Figure 21 for a neutral atmosphere $(H=0)$. Results are given for trailing edge noise only, turbulent inflow noise only and for the total noise. It appears that the maxima of overall SPL for the 3 curves are found upwind and downwind, and the minima are found crosswind $\left(90^{\circ} \pm 2^{\circ}\right.$ and $\left.270^{\circ} \pm 2^{\circ}\right)$. As already seen in Section 3, the AM strength is less than $1 \mathrm{~dB}$ in the upwind and downwind directions, and is maximum close to the crosswind direction, at slightly different directions for the three curves. The

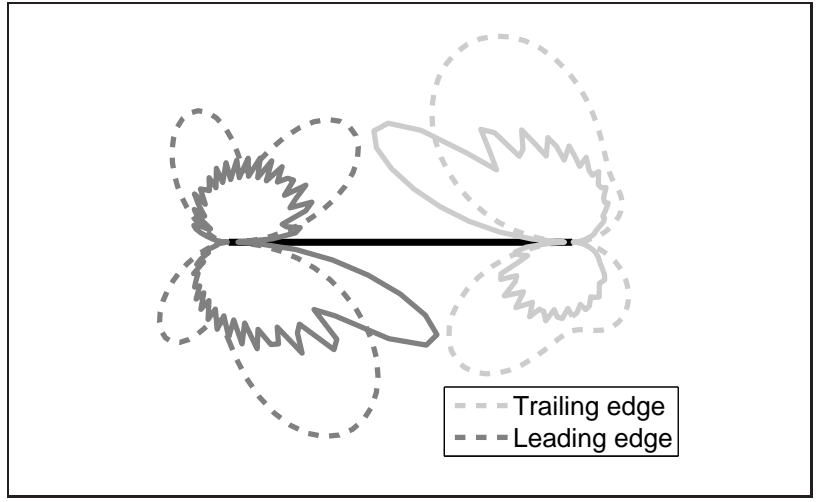

Figure 22: Normalized directivity of trailing edge noise and turbulent inflow noise for the tip segment. Dashed lines: $f=500 \mathrm{~Hz}$; solid lines: $f=4000 \mathrm{~Hz}$.

AM strength reaches a maximum of $10 \mathrm{~dB}$ a little upwind for trailing edge noise, of $9 \mathrm{~dB}$ a little downwind for turbulent inflow noise, and of only $4 \mathrm{~dB}$ exactly crosswind for the total noise.

To explain these differences, let us look first at the directivity of one blade segment in the coordinate system of the blade, as shown in Figure 22. Amiet's model predicts that trailing edge noise radiation is maximum towards the leading edge of the blade, while turbulent inflow noise radiation is maximum towards the trailing edge. The normalized directivity are frequency-dependent, with more lobes appearing with increasing frequency. This directivity pattern, as well as the twisting of the blade schematically represented in Figure 6, do explain that the directions where the minima are found are slightly different for the two noise mechanisms.

To better understand the directivity of AM strength, it is also useful to look at the variation of $S P L$ as a function of blade azimuthal position $\gamma$ shown in Figure 23 for directions $270^{\circ}$ and $278^{\circ}$. At $270^{\circ}$, exactly crosswind, trailing edge noise and turbulent inflow noise variations are in phase and their levels are comparable, which explains that the total noise follows the same trend with similar AM for the three curves. At $278^{\circ}$, slightly downwind, the situation is quite different with both mechanisms having out of phase variations and turbulent inflow noise levels being close to their minimum values. As a result, the total noise mostly follows the trailing edge noise variations and its $\mathrm{AM}$ strength is only $3 \mathrm{~dB}(\mathrm{~A})$, much smaller than the $9 \mathrm{~dB}(\mathrm{~A})$ obtained for turbulent inflow noise.

\section{Conclusion and future work}

In this paper, Amiet's analytical model for turbulent inflow noise and trailing edge noise is applied for compute wind turbine noise. First, we validated the model 


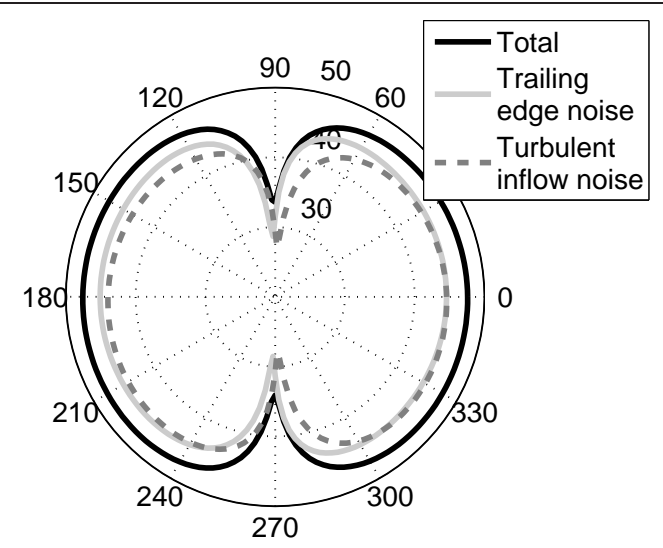

(a)

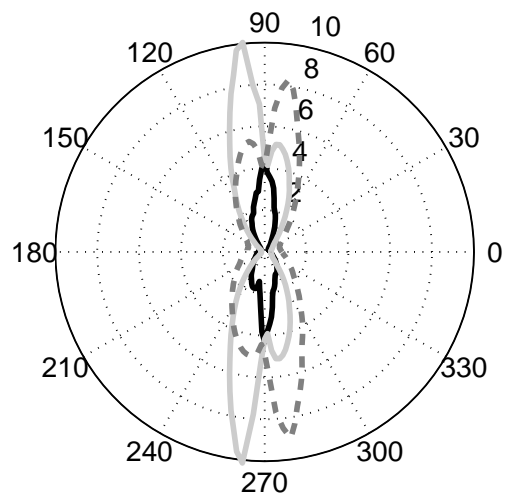

(b)

Figure 21: Directivity of (a) overall SPL and (b) amplitude modulation strength $100 \mathrm{~m}$ away from the wind turbine for case 2 and $H=0$ (neutral atmosphere).

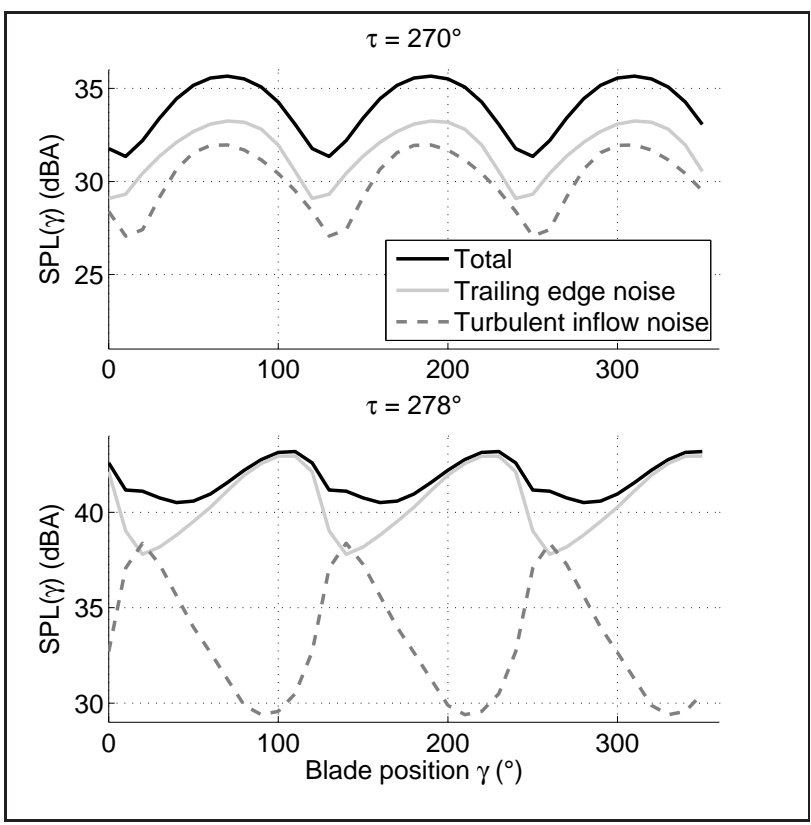

Figure 23: Amplitude modulation for trailing edge noise, turbulent inflow noise and for the overall noise at an horizontal angle of $270^{\circ}$ (top) and of $278^{\circ}$ (bottom) with respect to the wind direction, for case 2 and $H=0$.

predictions by comparison with wind tunnel experiments from the literature. We showed that trailing edge noise predictions are improved when the effect of an adverse pressure gradient is included. We also found that an empirical thickness correction for turbulent inflow noise may be considered to account for the reduction of noise level due to airfoil thickness.

Then, the model is adapted to rotating blades to predict wind turbine noise in the simple case where the wind speed is constant with height and turbu- lent inflow noise is neglected. Model predictions are compared to results from the literature for a $93 \mathrm{~m}$ diameter 2.3 MW wind turbine. The sound power level predictions are in good agreement with measurements at high frequencies when the APG model is used, but underestimate them at low frequencies. The predictions of directivity and amplitude modulation are also in agreement with results from the literature close to a wind turbine, with maximum SPL and minimum AM strength downwind and upwind, while minimum SPL and maximum AM strength are found in crosswind directions.

In the last part, we took into account wind shear and turbulence effects using the Monin-Obukhov similarity theory that is valid in the atmospheric surface layer over flat and homogeneous ground. On the one hand, we showed that wind shear causes variations of angle of attack that are largest in stable conditions (typically at night). Although the angle of attack variations due to wind shear produce a significant change in the wall pressure spectra at some blade segments, the increase in the trailing edge noise spectra at the receiver is almost negligible. On the other hand, turbulent inflow noise does vary significantly depending on atmospheric conditions. When both mechanisms are considered, SWL spectra are in much better agreement with measurements, with turbulent inflow noise dominating at low frequency (below $400 \mathrm{~Hz}$ approximately). Directivities of overall SPL and AM are similar for both mechanisms and for the total noise, with an $\mathrm{AM}$ strength that reaches at most $4 \mathrm{~dB}(\mathrm{~A})$ for the total noise, compared to up to $10 \mathrm{~dB}(\mathrm{~A})$ for each mechanism considered individually.

Several perspectives can be mentioned as a continuation of the present work. On the source side, it would be important to model separation/stall noise, that occurs when the AoA reaches large values. Recent studies have shown that this noise mechanism is 
a good candidate for explaining the enhanced amplitude modulation observed in the field [4]. Also, the effects of stronger wind shear and larger turbulence fluctuations could be studied, which would require to consider field measurements or theoretical tools that are more advanced than MOST. Finally, to be able to predict the noise perceived by potential neighbors at large distances from a wind turbine, we envisage to couple the present source model to a propagation model that takes into account atmospheric fluctuations such as the Parabolic Equation model [39].

\section{References}

[1] D. Bowdler, G. Leventhall: Wind turbine noise. Multi-Science Publishing, 2011.

[2] S. Oerlemans, J. G. Schepers: Prediction of wind turbine noise and validation against experiment. International Journal of Aeroacoustics 8 (2009) 555-584.

[3] M. Smith: Fundamental research into possible causes of amplitude modulation. - In: Wind Turbine Amplitude Modulation: Research to Improve Understanding as to its Cause and Effect. RenewableUK, 2013.

[4] S. Oerlemans: An explanation for enhanced amplitude modulation of wind turbine noise. - In: Wind Turbine Amplitude Modulation: Research to Improve Understanding as to its Cause and Effect. RenewableUK, 2013

[5] H. Madsen, A. Fischer, K. Kragh: Mechanisms and causes of amplitude modulation and other amplitude modulation of aeroacoustic wind turbine noise. - In: Wind Turbine Amplitude Modulation: Research to Improve Understanding as to its Cause and Effect. RenewableUK, 2013.

[6] G. van den Berg: Effects of the wind profile at night on wind turbine sound. Journal of Sound and Vibration 277 (2004) 955-970.

[7] K. Heutschi, R. Pieren, M. Müller, M. Manyoky, U. W. Hayek, K. Eggenschwiler: Auralization of wind turbine noise: Propagation filtering and vegetation noise synthesis. Acta Acustica united with Acustica 100 (2014) 13-24.

[8] T. Brooks, D. Pope, M. Marcolini: Airfoil self-noise and prediction. NASA Reference Publication 1218 (1989).

[9] W. Zhu, N. Heilskov, W. Shen, J. Sørensen: Modeling of aerodynamically generated noise from wind turbines. Journal of Solar Energy Engineering $\mathbf{1 2 7}$ (2005) 517-528.

[10] R. Amiet: Acoustic radiation from an airfoil in a turbulent stream. Journal of Sound and Vibration 41 (1975) 407-402.

[11] R. Amiet: Noise due to turbulent flow past a trailing edge. Journal of Sound and Vibration 47 (1976) 387393.

[12] S. Glegg, S. Baxter, A. Glendinning: The prediction of broadband noise from wind turbines. Journal of Sound and Vibration 118 (1987) 217-239.

[13] S. Lee, S. Lee, S. Lee: Numerical modeling of wind turbine aerodynamic noise in the time domain. Journal of the Acoustical Society of America 133 (2013) EL94-100.
[14] J. Casper, F. Farassat: Broadband trailing edge noise predictions in the time domain. Journal of Sound and Vibration 271 (2004) 159-176.

[15] M. Goody: Empirical spectral model of surface pressure fluctuations. AIAA Journal 42 (2004) 17881794.

[16] Y. Rozenberg, G. Robert, S. Moreau: Wall-pressure spectral model including the adverse pressure gradient effects. AIAA Journal 50 (2012) 2168-2179.

[17] M. Kamruzzaman, T. Lutz, W. Würz, W. Shen, W. Zhu, M. Hansen, F. Bertagnolio, H. Madsen: Validations and improvements of airfoil trailing edge noise prediction models using detailed experimental data. Wind Energy 15 (2012) 45-61.

[18] F. Bertagnolio, A. Fischer, W. Zhu: Tuning of turbulent boundary layer anisotropy for improved surface pressure and trailing-edge noise modeling. Journal of Sound and Vibration 333 (2014) 991-1010.

[19] W. Shen, W. Zhu, J. N. Sørensen: Aeroacoustic computations for turbulent airfoil flows. AIAA Journal 47 (2009) 1518-1527.

[20] M. Roger, S. Moreau: Extensions and limitations of analytical airfoil broadband noise models. International Journal of Acoustics 9 (2010) 273-305.

[21] W. Devenport, J. Staubs, S. Glegg: Sound radiation from real airfoils in turbulence. Journal of Sound and Vibration 329 (2010) 3470-3483.

[22] R. W. Paterson, R. K. Amiet: Acoustic radiation and surface pressure characteristics of an airfoil due to incident turbulence. NASA report, CR-2733 (1976).

[23] S. Glegg, W. Devenport: Panel methods for airfoils in turbulent flow. Journal of Sound and Vibration $\mathbf{3 2 9}$ (2010) 3709-3720.

[24] M. Roger, S. Moreau: Back-scattering correction and further extensions of amiet's trailing-edge noise model. part 1: theory. Journal of Sound and Vibration 286 (2005) 477-506.

[25] R. Amiet: Effect of the incident surface pressure field on noise due to turbulent flow past a trailing edge. Journal of Sound and Vibration 57 (1978) 305-306.

[26] M. Drela, M. Giles: Viscous-inviscid analysis of transonic and low reynolds number airfoils. AIAA Journal 25 (1987) 2168-2179.

[27] T. F. Brooks, T. H. Hodgson: Trailing edge noise prediction from measured surface pressures. Journal of Sound and Vibration 78 (1981) 69-117.

[28] R. Schlinker, R. K. Amiet: Helicopter rotor trailing edge noise. NASA report 3470 (1981).

[29] V. P. Blandeau, P. F. Joseph: Validation of amiet's model for propeller trailing-edge noise. AIAA Journal 49 (2011) 1057-1066.

[30] S. Sinayoko, M. Kingan, A. Agarwal: Trailing edge noise theory for rotating blades in uniform flow. Proc. R. Soc. A 469 (2013) 20130065.

[31] S. Oerlemans, P. Sijtsma, B. M. López: Location and quantification of noise sources on a wind turbine. Journal of Sound and Vibration (2007) 869-883.

[32] G. Leloudas: Optimization of wind turbines with respect to noise. Diploma Thesis. DTU, 2006.

[33] A. Creech, W.-G. Früh, A. Maguire: Simulations of an offshore wind farm using large eddy simulation and a torque-controlled actuator disc model. Surveys in Geophysics (2015) 427-481. 
[34] D. Wilson: The sound-speed gradient and refraction in the near-ground atmosphere. J. Acoust. Soc. Am. 113 (2003) 750-757.

[35] T. Foken: 50 years of the monin-obukhov similarity theory. Boundary-Layer Meteorology 119 (2006) 431-447.

[36] S. Cheinet: A numerical approach to sound levels in near-surface refractive shadows. J. Acoust. Soc. Am. 131 (2012) 1946-1958.

[37] E. Salomons: Computational atmospheric acoustics. Kluwer Academic Publishers, 2001.

[38] V. Ostashev, D. Wilson: Relative contributions from temperature and wind velocity fluctuations to the statistical moments of a sound field in a turbulent atmosphere. Acta Acustica united with Acustica 86 (2000) 260-268.

[39] B. Cotté, P. Blanc-Benon: Estimates of the relevant turbulent scales for acoustic propagation in an upward refracting atmosphere. Acta Acustica united with Acustica 93 (2007) 944-958.

\section{A. Expressions for the velocity pro- files and atmospheric turbulence parameters}

\section{A.1. Velocity profiles}

The mean velocity profile $U(z)$ given in Equation (12) involves a function $\psi_{u}$ that depends on the stability of the atmosphere. For an unstable atmosphere $\left(L_{*}<\right.$ 0) $[37,34]$ :

$$
\begin{aligned}
\psi_{u}=2 \ln \left(\frac{1+x}{2}\right)+\ln \left(\frac{1+x^{2}}{2}\right) \\
\quad-2 \arctan x+\frac{\pi}{2},
\end{aligned}
$$

with $x=\left(1-16 z / L_{*}\right)^{1 / 4}$. For a stable atmosphere $\left(L_{*}>0\right)[37,34]$ :

$$
\begin{aligned}
& \psi_{w}= \\
& \left\{\begin{array}{l}
-5 z / L_{*} \text { for } z / L_{*} \leq 0.5 \\
-7 \ln \left(z / L_{*}\right)-\frac{4.25}{z / L_{*}}+\frac{0.5}{\left(z / L_{*}\right)^{2}}-0.852 \quad \text { elsewhere. }
\end{array}\right.
\end{aligned}
$$

\section{A.2. Atmospheric turbulence parameters}

We detail in this section the expressions for the heightdependent standard deviation of turbulent velocity fluctuations $\sigma_{u}$ and integral length scale $\Lambda$ appearing in the von Kármán spectrum. Cheinet [36] gives the following empirical relationships for the variance $\sigma_{u}^{2}$ :

$$
\sigma_{u}^{2}=\left\{\begin{array}{l}
u_{*}^{2}\left[\alpha_{1}+\frac{1}{\left|L_{*}\right|}\left(\alpha_{2} z_{i}+\alpha_{3} z\right)\right]^{2 / 3} \quad \text { if } \quad L_{*}<0, \\
u_{*}^{2}\left[1.73+3.3\left(\frac{z}{L_{*}}\right)^{0.5}\right]^{2} \quad \text { if } \quad L_{*}>0,
\end{array}\right.
$$

with $z_{i}$ the mixed layer height (set to $1000 \mathrm{~m}$ ), $\alpha_{1}=$ 5.2, $\alpha_{2}=0.52$, and $\alpha_{3}=0$ in the surface layer $\left(z \leq 0.1 z_{i}\right)$. Both expressions yield $\sigma_{u}^{2}=3.0 u_{*}^{2}$ when $L_{*} \rightarrow \infty$ (neutral conditions).

The integral length scale $\Lambda=L_{\text {outer }} / 1.339$, with $L_{\text {outer }}$ the outer scale in the von Kármán model given by [36]:

$$
L_{\text {outer }}=\left(1.91 \frac{\sigma_{u}^{2}}{C_{u}^{2}}\right)^{3 / 2} .
$$

$C_{u}^{2}$ is the structure parameter of momentum fluctuations parametrized as follows:

$$
C_{u}^{2}=\frac{u_{*}^{2}}{z^{2 / 3}} f_{u}\left(\frac{z}{L_{*}}\right),
$$

where

$$
f_{u}(\xi)=\left\{\begin{array}{l}
3.9\left(\frac{1-\xi}{1-7 \xi}-\xi\right)^{2 / 3} \quad \text { for } \quad \xi \leq 0 \\
3.9(1+5 \xi)^{2 / 3} \quad \text { for } \quad \xi>0
\end{array}\right.
$$

with $\xi=z / L_{*}$. Both expressions yield $L_{\text {outer }}=1.8 z$ for purely shear-driven turbulence, i.e. for $\xi=0$ (neutral conditions). 